\title{
DIFFRACTION AT COLLIDER ENERGIES
}

L. L. Frankfurt

Department of Physics, FM-15

University of Washington, Seattle, WA 98195
DCE/ER/40427--33-N92

DE93 010200

Talk presented at the XXVI International Conference on High Energy Physics

Dallas, Texas, August 1992

\section{PREPARED FOR THE U.S. DEPARTMENT OF ENERGY}

This report was prepared as an account of work sponsored by the United States Government. Neither the United States nor the United States Department of Energy, nor any of their employees, nor any of their contractors, subcontractors, or their employees, makes any warranty, express or implied, or assumes any legal liability or responsibility for the product or process disclosed, or represents that its use would not infringe privately-owned rights.

By acceptance of this article, the publisher and/or recipient acknowledges the U.S. Government's right to retain a nonexclusive, royalty-free license in and to any copyright covering this paper. 


\title{
DIFFRACTION AT COLLIDER ENERGIES
}

\author{
L. L. Frankfurt \\ Department of Physics, FM-15 \\ University of Washington \\ Seattle, Washington 98195
}

\begin{abstract}
The aim of this talk is to outline lessons with "soft" hadron physics to explain a) feasibility to observe and to investigate color transparency, color opacity effects at colliders; b) significant probability and specific features of hard diffractive processes; c) feasibility to investigate components of parton wave functions of hadrons with minimal number of constituents. This new physies would be more important with increase of collision energy.
\end{abstract}

\section{"SOFT" HADRON PHYSICS}

i) Pomeron exchange with the trajectory $\alpha(t)=1.08+0.2 t$ provides a remarkable first approximation to near forward elastic scattering from ISR to Tevatron (ci. discussion in Ref. 1). Odderon exchange may help ${ }^{2}$ to explain anomalously large value of

$\operatorname{Re} A p \bar{p} / \operatorname{Im} A \bar{p} p / t=0$ observed by UA (4).

ii) Supercritical pomeron $(\alpha(0)>1)$ reasonably describes energy dependence of elastic cross sections of $p p, \bar{p} p$ scattering within forward peak and of total cross sections (cf. Refs. 1,4 and references therein).

iii) Inelastic intermediate states (diffractive dissociation of initial hadrons) should be included into eikonal approximation. ${ }^{5}$

iv) ISR data on single diffraction can be reasonably described by triple pomeron formulae. ${ }^{6}$ If so, Reggeon Calculus predicts more narrow distribution in multiplicities for central cluster $X$ than in $p p$ scattering due to smallness of triple pomeron vertex. At the same time UA(1) data for the double diffraction: $\bar{p} p \rightarrow X_{1}+X+X_{2}$ found too fast increase of multiplicity with $M_{X}$ for central cluster $X$. Presence of trigger for $E_{T}>3$ $\mathrm{GeV}$ which effectively suppresses the contribution of small masses makes theoretical interpretation difficult.

Observed dependence of total and elastic cross sections on initial energy is a temporary phenomenon. It should be changed to avoid contradiction with unitarity condition that partial waves cannot exceed 1 . Really partial waves of $\bar{p} p$ collision with impact parameter $b=0$ extracted from Tevatron data on total and elastic cross sections are $|f| b=0) \mid=1 / 2$ and increase with energy.

\section{COLOR COHERENT EFFECTS}

For sufficiently large projectile energies the length of spatial transition between different quark-gluon configurations $|n\rangle$ in the wave function of projectile hadron $h$ exceeds $r_{T}$ - the radius of a target $T$. Really uncertainty principle shows that: 


$$
\begin{aligned}
& 1 /(E n-E h) \approx \\
& 2 E_{h} /\left[\sum_{i}\left(m_{i}^{2}+k_{i t}^{2}\right) / \alpha_{i}-m_{h}^{2}\right]>r_{T}
\end{aligned}
$$

In the eikonal approximation Eq. (1) means that due to the large Lorentz factor orbits of constituents in the fast hadron $h$ are frozen during collision and the total cross section $\sigma_{h T}$ becomes almost incoherent sum of cross sections for the different configurations $|n\rangle$. In $\mathrm{pQCD}^{8}$ and in nonperturbative regime ${ }^{9}$ different configurations have different cross sections depending on its transverse radius. So the fundamental quantity is $F_{h}(\sigma)$ - the distribution over the value of cross section. Such a quantity formally arises in the formalism of scattering states. ${ }^{10}$

Four moments of $P_{h}(\sigma)-\left\langle\sigma^{n}\right\rangle \equiv$ $\int P(\sigma) \sigma^{n} d \sigma$ were extracted from experimental data. $\left\langle\sigma^{0}\right\rangle$ is conservation of probability; $\left\langle\sigma^{2}\right\rangle$ is average cross section. $\left\langle\sigma^{(2)}\right\rangle$ is extracted frorn single diffraction in Ref. 11 and from inelastic shadowing correction to $\sigma_{p d}^{\text {tot }}$ in Ref. 12. $\left\langle\sigma^{3}\right\rangle$ has been extracted from liffractive dissociation of proton of deuteron. ${ }^{12}$ The distribution Eq. (12) reproduces all these momenta ${ }^{12}$ :

$$
P_{N}(\sigma)=0.66\left(\sigma /(\sigma+a) \exp -\left(\frac{\sigma-\sigma_{0}}{1.1 \sigma_{0}}\right)^{6}\right.
$$

where $\sigma_{0}=0.89\langle\sigma\rangle ; a=0.1 \sigma_{0}$. Note that $P_{N}(\sigma) \sim \sigma$ at $\sigma \rightarrow 0$ since a nucleon is a system of 3 quarks. ${ }^{17}$

\section{New Phenomena at Colliders}

1. Significant probability of $P_{N}(\sigma \ll$ $\langle\sigma\rangle)$ leads to numerous color transparency (ct) phenomena and gives possibility to probe QCD in a new way (see review ${ }^{16}$ ). The most striking example is the significant transparency for spectator nucleons in the central nucleusnucleus collisions. This physics may reveal itself starting from CERiN nuclear beam energies. ${ }^{17}$

2. Analysis performed in Ref. (15) shows that ct phenomena suggested for a nucleon projectile (cf. review ${ }^{16}$ ) should be much more pronounced for the pion piojectile. This is physics for FNAL, KAON.

3. Large $(\sim 1 / 2)$ probability of $P_{N}(\sigma)$ for $\sigma>\langle\sigma\rangle$ leads to numerous color opacity phenomena:

i) Broadening of large $E_{T}$ tail seems to be observed by NA34 in heavy ion collisions. ${ }^{18}$

ii) Percolation phase transitions and peculiar fractals are expected in central heavy ion collisions. ${ }^{19}$ Use of this phenomenon may help to search for other phase transitions.

\section{HARD DIFFRACTIVE PROCESSES}

One of the hard processes relevant for $P(\sigma \rightarrow 0)$ is the single diffractive dissociation: $\bar{p}+p_{i} \rightarrow$ “ $M_{X}$ " $+p_{f}$ in the kinematics

$$
\left|M_{X}^{2} / s\right| \ll 1,\left|\left[\left(P_{i}-P_{f}\right)^{2}=t\right] / s\right| \ll 1 .
$$

Requirement of large $p_{t}$ trigger selects small spatial size configuration in the initial (final) nucleons. Three phenomena work in the same direction. i) radiation is kinematically suppressed ii) for collision of black bodies diffraction is small. So the contribution of configurations with large cross section is suppressed. iii) Analysis of electromagnetic form factors of hadrons in realistic models of hadrons ${ }^{20}$ shows that color screening phenomenon may reveal itself for $Q^{2}>1-2 \mathrm{GeV}^{2}$. So there is a good chance for the applicability of pQCD in the single diffraction at $|t| \geq 1 \mathrm{GeV}^{2}$. In pQCD for small $\alpha_{s}$ cross section is given by gluon ladder and has the form: ${ }^{21}$ 


$$
\begin{gathered}
\frac{d \sigma p \bar{p}}{d t d M_{X}^{2}} \rightarrow{ }^{"} M_{x}{ }^{n}+p=\alpha_{S}^{2}(t) \frac{\left(\frac{S}{M_{X}^{2}}\right)^{2 w}}{\alpha_{S}^{2} \ln ^{3}\left(s / M_{X}^{2}\right)} \\
1 / M_{X}^{2}\left|\frac{t}{M_{X}^{2}}\right| G_{N}\left(\frac{(t)}{M_{X}^{2}},|t|\right)\left|F_{\mid P N}^{g}(t)\right|^{2}
\end{gathered}
$$

Here $w(t)=12 \alpha_{s}(t) \ln 2 / \pi$ and $F_{P N}$ is the two gluon form factor of a nucleon. Eq. (4) is a striking prediction of $\mathrm{pQCD}$. Really soft physics predicts that the peak at $x=1$ and $t=0\left(M_{X}^{2} / S=1-x\right)$ should soften with the increase of $t$. But Eq. (4) means that the peak at $x \rightarrow 1$ should become even more pronounced at larger $t$.

We want to draw attention that in the eikonal approximation (soft physics) in difference from $\mathrm{pQCD}$ the role of multipomeron exchanges increases with increase of $t$ (cf. Ref. 22).

At large $t$ where pQCD dominate one can calculate diffractive production of high $p_{t}$ jets:

$$
p+p \rightarrow\left\{\text { jet }_{1}+\text { jet }_{2}+X\right\}+p
$$

Distinctive prediction of $\mathrm{pQCD}$ is that gluon structure function of the pomeron at large $z$

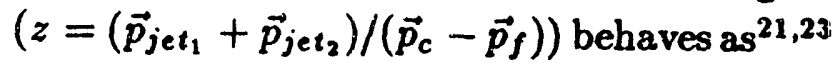

$$
z G_{\mid p}(z) \sim \frac{1}{1-z}, \delta(1-z)
$$

This prediction should be confronted with predictions for the nonperturbative pomeron:

$$
z G_{l p}(z) \sim(1-z)^{n} \text { with } n=1-5
$$

(cf. Ref. 24 and refs. therein). Thus in pQCD pomeron behaves like point-like particle.

Recent UA(8) data found that in $30 \%$ of events diiets carry the whole momen'um of the pomeron. ${ }^{25}$

\section{REFERENCES}

1. M.M. Block and A. White " 4 th Blois Workshop on Elastic and Diffractive Scattering", Nucl. Phys. B. (Proc. Suppl.) 25, (1092).

2. P. Cauron and B. Nicolescu, "The Importance of the Measurement of $\rho$ for the understanding of the High Energy Hadron-Hadron Scattering," Contributed paper N154.

3. D. Berhard et al., UA4 Collaboration, p. 4, B198, 583 (1987).

4. E. Gottsman et al. "A Quantitative Description of the Pomeron", Contributed paper $\mathrm{N} 48$.

5. R. Engel et. al. "Extrapolation of Hadron Cross Sections to Collider Energies within the Two Component Dual Parton Model", Contributed paper N277.

6. K. Coulianos, Phys Rep. 11, 169 (1983).

7. D. Joyce, et al. "Double Pomeron Exchange in $\bar{p} p$ Interactions at $0.63 \mathrm{TeV}$, Contributed paper N229.

8. F.E. Low, Phys. Rev. D12, 163, (1975) S. Nussinov, Phys. Rev. Lett. 34, 1286 (1975), Phys. Rev. D14, 246, (1976).

9. L.L. Frankfurt and M.I. Strikman, Phys. Rep. 160, 235 (1988).

10. E.L Feinberg and I.V. Pomeranchuk, Suppl. Nuovo Cimento. 111,652,(1956). M.I. Good and W.A. Walker, Phys. Rev. $120,1857,(1960)$.

11. H. Miettinen and J. Pumplin, Phys. Rev. D18, 1696, (1978), Phys. Rev. Lett. 42, 204, (1979).

12. B. Blättel et al., "Cross-Section Fluctuations," Contributed paper, Nucl. Phys. A. 544, 479, (1992).

13. C. Bertch et al., Phys. Rev. Lett. 47, 297, (1981). 
14. L.L. Frankfurt, to be published.

15. B. Blättel, et al., to be published, Contributed papers 650,682 .

16. L. Frankfurt and M. Strikman, Prog. Part. Nucl. Phys., 27, 135, (1991).

17. L. Frankfurt and M. Strikman, Phys. Rev. Lett., 66, 2289, (1991).

18. H. Heiselberg, et. al., Phys. Rev. Lett., 67, 2946, (1991).

19. A. Bulgac and L. Frankfurt, to be published.
20. L. Frankfurt et al., "Comment for Particle and Nuclear Physics", September, 1992.

21. L. Frankfurt and M. Strikman, Phys. Rev. Lett., 63, 1914, (1989).

22. V. Abramovsky and R. Betman, Contributed paper N763.

23. L. Frankfurt, Talk at FAD Meeting, Dallas, March, 1992.

24. G. Ingelman and P. Schlein, Phys. Lett 152B, 256, (1985).

25. P. Schlein, Comment after Bartke talk at this Conference. 

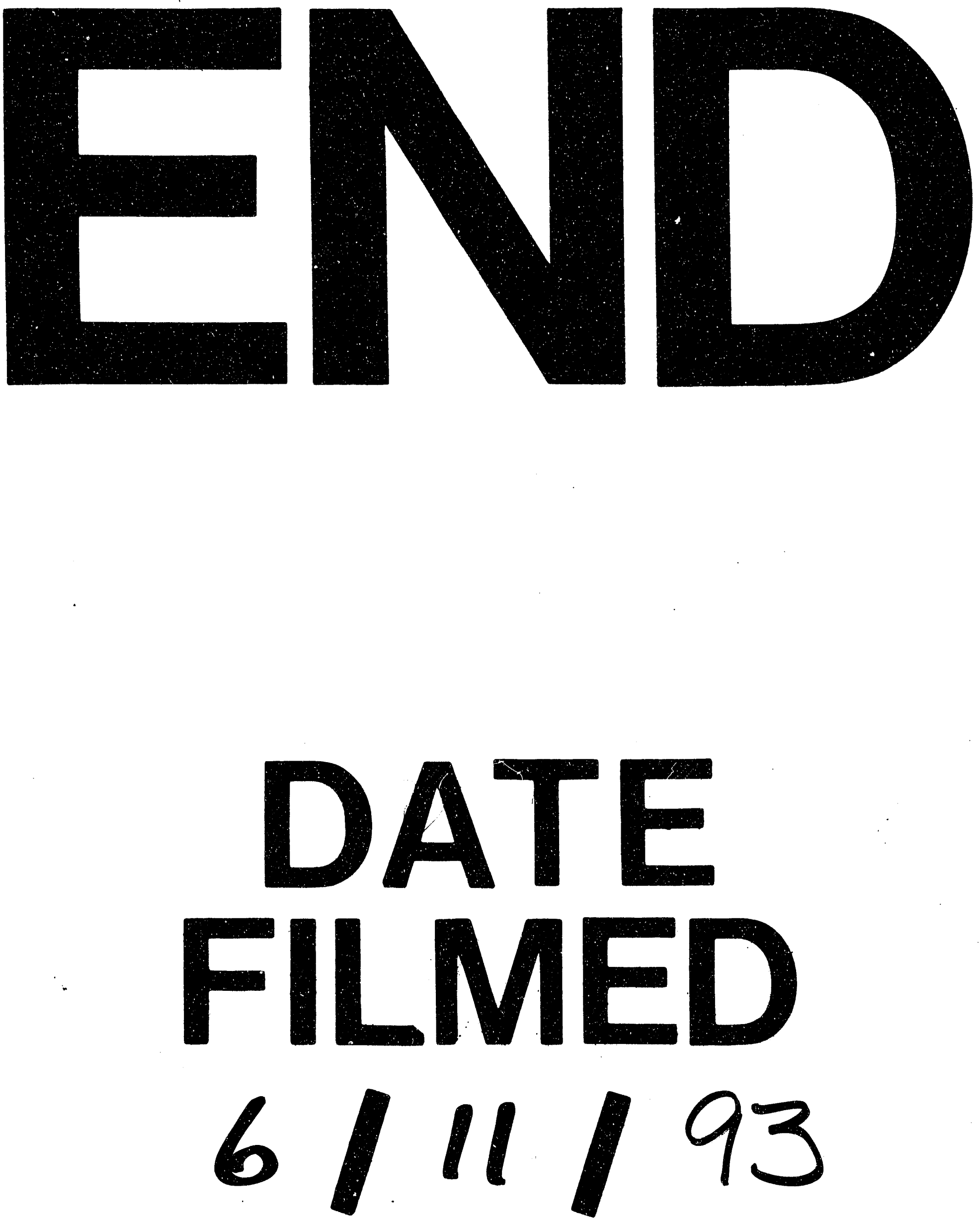
